\title{
Bioequivalence of Two Co-formulations of Emtricitabine/Tenofovir Alafenamide Fixed-Dose Combinations with 200/10 mg and 200/25 mg
}

Zack J, Chu H, Chuck S*, Rhee M, Koziara J, West S, Fang L and Kearney B

Gilead Sciences Inc, Foster City, CA, United States

\begin{abstract}
Emtricitabine/tenofovir alafenamide (FTC/TAF) is the next advancement in nucleotide reverse transcriptase inhibitor (NRTI) backbone for the treatment of HIV-1 in adults. Tenofovir disoproxil fumarate (TDF), an oral prodrug of tenofovir (TFV), is a preferred NRTI, but it is also associated with nephrotoxicity and reduced bone mineral density (BMD). TDF has been replaced by TAF, a novel, prodrug of TFV with a unique metabolic pathway leading to $>90 \%$ lower circulating plasma of TFV. Less nephrotoxicity, less BMD reduction in treatment-naïve subjects, and BMD increase in virologically suppressed patients on TAF in clinical trials. The two studies presented here were conducted in healthy subjects. Study 1472 evaluated the pharmacokinetics (PK) and bioequivalence (BE) of the components of FTC/TAF 200/10 mg fixed-dose combination (FDC) administered with elvitegravir $150 \mathrm{mg}$ and cobicistat $150 \mathrm{mg}$ tablets to elvitegravir (E)/cobicistat (C)/FTC (F)/TAF (150/150/200/10 mg) single-tablet regimen (STR). Study 1473 evaluated the PK and BE of the components of FTC/TAF 200/25 FDC to E/C/F/TAF STR. One-hundred and 116 subjects were randomized into the single-dose, open-label, 2-way, crossover Phase 1 Study 1472 and Study 1473 , respectively; two subjects did not complete Study 1472. Serial blood samples were obtained over 144 hours after administration of each treatment, and pharmacokinetic parameters were calculated. Formulation bioequivalence was assessed by $90 \%$ confidence intervals (Cls) for the geometric least-squares mean (GLSM) ratios of pharmacokinetic parameter $A \cup C_{\text {last }}, A U C_{\text {inf }}$, and $C_{\text {max }}$ for each component of FTC/TAF FDC to FTC and TAF administered as E/C/F/ TAF STR. The test and reference treatments were generally well tolerated. The $90 \% \mathrm{Cls}$ for the GLSM ratios of the primary pharmacokinetic parameter $A \cup C_{\text {last }}, A \cup C_{\text {inf, }}$ and $C_{\text {max }}$ for test versus reference treatments were within the protocol-specified bioequivalence boundary of $80 \%$ to $125 \%$ for FTC and TAF. FTC/TAF 200/10 mg and 200/25 mg are the next advancement in NRTI backbone for treatment of HIV-1 adults and adolescents, with an emphasis on safety while maintaining the efficacy and convenience of its predecessor.
\end{abstract}

Keywords: Bioequivalence; Tenofovir alafenamide; Emtricitabine; Pharmacokinetics; Fixed-dose combination; HIV-1; TAF

\section{Introduction}

Tenofovir disoproxil fumarate (TDF), a prodrug of tenofovir (TFV), is the nucleotide reverse transcriptase inhibitor (NRTI) in the majority of Department of Health and Human Services (DHHS) recommended regimens for antiviral agents in HIV-1-infected adults and adolescents [1]. However, TDF is associated with nephrotoxicity and reduced bone mineral density (BMD) [1,2]. Tenofovir alafenamide (TAF) is a novel prodrug of TFV. TAF has been selected for clinical development because it has a different metabolic pathway from TDF that enhances lymphocyte delivery of TFV; this unique metabolic pathway results in 91\% lower circulating plasma concentrations of TFV while maintaining similar intracellular levels of the active phosphorylated metabolite TFVdiphosphate (TFV-DP) when TAF $10 \mathrm{mg}$ is administered as elvitegravir (EVG; E)/cobicistat (COBI; C)/FTC/TAF (E/C/F/TAF) [3]. In a Phase 1b study, there were $97 \%$ and $86 \%$ lower circulating plasma concentrations of TFV with TAF $8 \mathrm{mg}$ and $25 \mathrm{mg}$, respectively, than TDF $300 \mathrm{mg}$ [4]. Due to lower TFV exposure, TAF has demonstrated less nephrotoxicity, less bone mineral density (BMD) decreases in treatment-naïve patients, and $\mathrm{BMD}$ increases in virologically suppressed patients in pivotal trials of TAF-containing single-tablet regimen (STR) of E/C/F/TAF when compared to TDF-containing regimens. TAF has been co-formulated with FTC, referred to as F/TAF, and if approved, will allow clinicians to combine F/TAF with other antiretroviral (ARV) agents. Furthermore, a fixed-dose combination (FDC) reduces the pill burden, an important consideration to improve adherence [1].

The recommended TAF dose (10 or $25 \mathrm{mg}$ ) is based on whether or not the coadministered third agent has any clinically relevant effect on TAF exposures. This ensures that patients have a TAF systemic exposure that is within the range of the reference exposure achieved with TAF $25 \mathrm{mg}$ or with TAF $10 \mathrm{mg}$ when administered as $\mathrm{E} / \mathrm{C} / \mathrm{F} /$ TAF. Pharmacokinetic (PK) enhancers, like COBI and ritonavir (RTV), substantially increase TAF exposure by inhibiting intestinal p-glycoprotein-mediated efflux of TAF [5-9]. Thus, the TAF $10 \mathrm{mg}$ dose is utilized with RTV/COBI-boosted regimens, and TAF $25 \mathrm{mg}$ dose is utilized with unboosted regimens.

The two studies presented here were conducted to establish the bioequivalence (PK comparability) between F/TAF 200/10 mg (Study 1472) and F/TAF 200/25 mg (Study 1473) and the reference product of $\mathrm{E} / \mathrm{C} / \mathrm{F} / \mathrm{TAF}$, which is supported by extensive clinical safety and efficacy data. E/C/F/TAF has been approved by US and EU Regulatory Authorities and has been designated a recommended regimen for treatment-naïve and virologically suppressed individuals in the United States.

\section{Methods}

\section{Study population}

Healthy male and nonpregnant, nonlactating female subjects,

*Corresponding author: Chuck S, Gilead Sciences Inc, Foster City, CA, United States, Tel: (650)522-3000; (650)522-5801; E-mail: susan.chuck@gilead.com

Received December 21, 2015; Accepted January 22, 2016; Published January 29, 2016

Citation: Zack J, Chu H, Chuck S, Rhee M, Koziara J, et al. (2016) Bioequivalence of Two Co-formulations of Emtricitabine/Tenofovir Alafenamide Fixed-Dose Combinations with 200/10 mg and 200/25 mg. J Bioequiv Availab 8: 068-073. doi:10.4172/jbb.1000270

Copyright: (c) 2016 Zack J, et al. This is an open-access article distributed under the terms of the Creative Commons Attribution License, which permits unrestricted use, distribution, and reproduction in any medium, provided the original author and source are credited. 
between 18 and 45 years of age (inclusive), with a body mass index (BMI) between 19 and $30 \mathrm{~kg} / \mathrm{m}^{2}$ (inclusive), in general good health, and a creatinine clearance $\geq 70 \mathrm{~mL} /$ minute (using the Cockcroft-Gault formula and actual body weight) were enrolled in the F/TAF 200/10 $\mathrm{mg}(\mathrm{N}=100)$ and $\mathrm{F} / \mathrm{TAF} 200 / 25 \mathrm{mg}(\mathrm{N}=116)$ bioequivalence studies, respectively.

A negative serum pregnancy test was required for female subjects of childbearing potential. Screening laboratory evaluations (hematology, chemistry, and urinalysis) had to be within the normal range. Inclusion criteria included having a normal 12-lead electrocardiogram (ECG). Subjects who had a history of recurring syncope, palpitations, or recurring, unexplained dizziness; who had an implanted defibrillator or pacemaker; or who had any serious or active medical or psychiatric illness were excluded. They were also excluded if they took any prescription medications or over-the-counter medications including herbal products within 28 days of commencing study drug dosing; exceptions were vitamins, acetaminophen, ibuprofen, and/or hormonal contraceptive medications. Additionally, subjects treated with systemic steroids, immunosuppressant therapies, or chemotherapeutic agents within 3 months of study screening were excluded. Subjects with current alcohol or substance abuse that could potentially interfere with compliance, as judged by the investigator, were excluded. Subjects were restricted, both before and through discharge, from consuming alcohol-containing products; using nicotine-containing products; and consuming grapefruit juice, grapefruits, and Seville orange juice. Consuming caffeine and other methyl-xanthines-containing products were prohibited only on dosing days.

Informed consent was obtained from each subject before initiation of study procedures. The protocol was reviewed and approved by a central institutional review board (Schulman and Associates, Research Triangle Park, NC). The study was performed in accordance with the principles of the Declaration of Helsinki and adhered to the basic principles of Good Clinical Practice as outlined in the US Code of Federal Regulations (CFR) Title 21, Part 312.

\section{Study methods}

Both studies were randomized, single-dose, open-label, 2-way, crossover Phase 1 studies in healthy adults under fed conditions (moderate calorie/ moderate fat meal) after an overnight fast for at least 10 hours. In the F/TAF 200/10 mg bioequivalence study, Treatment A contained a $200 \mathrm{mg}$ of FTC and $10 \mathrm{mg}$ of TAF FDC tablet administered simultaneously with elvitegravir (EVG) $150 \mathrm{mg}$ and cobicistat (COBI) $150 \mathrm{mg}$ tablets orally; Treatment B contained a $150 \mathrm{mg}$ of EVG, $150 \mathrm{mg}$ of COBI, $200 \mathrm{mg}$ of FTC, and $10 \mathrm{mg}$ of TAF (EVG/COBI/FTC/TAF) STR. In the F/TAF 200/25 mg bioequivalence study, Treatment A was a F/TAF 200/25 mg FDC tablet; Treatment B was an EVG/COBI/FTC/ TAF (150/150/200/10 mg) STR.

Subjects were randomized to 1 of 2 treatment sequences $(A B$, BA). A single dose of study drug(s) was administered on Days 1 and 7 within 5 minutes of completing a standardized moderate fat/moderate calorie breakfast (approximately 600 calories and approximately $27 \%$ fat). Following study drug administration, subjects were restricted from food intake until after the 4-hour PK blood sampling time point. Subjects were discharged on Day 13, with a follow-up phone call on Day $21( \pm 2)$.

Serial blood samples for PK assessments were collected at the following time points: 0 (predose), 5 minutes, 15 minutes, $0.5,0.75,1$, $1.5,2,3,4,5,8,12,24,48,72,96,120$, and 144 hours after administration of each treatment. The samples at the $144^{\text {th }}$ hour were collected predose on Day 7. The number, frequency, and timing of blood samples were based on the concentration-time profiles of the individual drugs to assess bioequivalence accurately based on maximum concentration $\left(\mathrm{C}_{\max }\right)$ and area under the concentration-time curve (AUC).

\section{Bioanalytic methods}

Concentrations of FTC and TAF in human plasma samples were determined using fully validated high-performance liquid chromatography-tandem mass spectroscopy (LC/MS/MS) bioanalytical methods. All samples were analyzed within the timeframe supported by frozen stability storage data. Briefly, the methodology for FTC and TAF was as follows: $100 \mu \mathrm{L}$ and $50 \mu \mathrm{L}$ of human plasma were spiked with internal standards $\left[{ }^{13} \mathrm{C}_{1}\right],\left[{ }^{15} \mathrm{~N}_{2}\right]$-emtricitabine and $\left[{ }^{2} \mathrm{H}_{7}\right]$-GS-7171 (tenofovir derivative), respectively. The FTC sample was then processed by protein precipitation with methanol. The TAF sample was then processed by protein precipitation with acetonitrile-formic acid. After this processing, the organic solvent was evaporated, and an aliquot of the reconstituted sample extract was injected into the LC/MS/MS system.

For each method, the results of within-run (intra-assay) and between-run (inter-assay) precision assessments were reported as the coefficients of variation, each expressed as $\% \mathrm{CV}$, and the results of accuracy assessments were reported as the relative error values expressed as \%RE. For FTC, the calibrated range of the method was 5 to $3000 \mathrm{ng} / \mathrm{mL}$; all $\% \mathrm{CV}$ values were $<8.6 \%$ and all $\% \mathrm{RE}$ values were within $\pm 9.8 \%$ of $100 \%$. For TAF, the calibrated range of the method was 1 to $1000 \mathrm{ng} / \mathrm{mL}$; all \%CV values were $<9.6 \%$ and all $\% \mathrm{RE}$ values were within $\pm 7.8 \%$ of $100 \%$.

\section{Safety assessments}

Safety was evaluated throughout each study and included physical examination, vital sign measurement, clinical laboratory test, evaluation of adverse events (AEs), and review of concomitant medications. Treatment-emergent AEs were defined as any AEs with an onset date of on or after the date of the first dose of study drug and on or before the date of the last dose of study drug plus 30 days, or any AEs that led to premature discontinuation of study drug. Adverse events were coded using the Medical Dictionary for Regulatory Activities (MedDRA), version 17.0. The severity of AEs and laboratory abnormalities was graded according to the Gilead Sciences, Inc. (GSI) Grading Scale for Severity of Adverse Events and Laboratory Abnormalities (grades 1-4).

\section{Pharmacokinetic analysis}

The PK analysis sets for FTC and TAF included all randomized subjects who received at least 1 dose of study drug and had at least 1 plasma concentration data reported by the PK lab. Samples below the limit of quantification (BLQ) of bioanalytical assays that were taken before study drug administration were given a value of 0 to prevent overestimation of the initial AUC. For AUC, samples that are BLQ at all other time points were treated as missing data in WinNonlin. For summary statistics, values that are BLQ at postdose time points were treated as one-half the value of the lower limit of quantitation (LLOQ). Furthermore, subjects with predose concentration values $>5 \%$ of the maximum observed plasma concentration of drug $\left(\mathrm{C}_{\max }\right)$ for either period were excluded from the corresponding $\mathrm{PK}$ analysis set.

PK parameters were estimated using standard of noncompartmental methods [Phoenix WinNonlin, version 6.4; Certara USA, Inc., Princeton, NJ] from the plasma concentration-time data 
Citation: Zack J, Chu H, Chuck S, Rhee M, Koziara J, et al. (2016) Bioequivalence of Two Co-formulations of Emtricitabine/Tenofovir Alafenamide Fixed-Dose Combinations with 200/10 mg and 200/25 mg. J Bioequiv Availab 8: 068-073. doi:10.4172/jbb.1000270

of FTC and TAF after administration of a single dose(s) of test and reference treatments. The primary PK parameters are area under the plasma concentration versus time curve extrapolated to infinity $\left(\mathrm{AUC}_{\text {inf }}\right)$, area under the plasma concentration versus time curve from time 0 to the last quantifiable concentration $\left(\mathrm{AUC}_{\text {last }}\right)$, and $\mathrm{C}_{\max }$ of FTC and TAF. The following PK parameters were calculated for FTC, TAF, COBI, and EVG: $\mathrm{AUC}_{\text {inf }}, \mathrm{AUC}_{\text {last }}$, half-life $\left(\mathrm{t}_{1 / 2}\right), \mathrm{C}_{\text {max }}$, and time to maximum concentration $\left(\mathrm{T}_{\max }\right)$.

\section{Statistical analysis}

The primary endpoints were the $\mathrm{PK}$ parameters $\mathrm{AUC}_{\text {last }}, \mathrm{AUC}_{\text {inf }}$ and $\mathrm{C}_{\max }$ of FTC and TAF. Bioequivalence of FTC and TAF in the F/ TAF FDC to the FTC and TAF components in E/C/F/TAF STR was concluded if the $90 \%$ confidence interval (CI) of the geometric leastsquares mean (GLSM) ratio of the PK parameters for each analyte between the 2 formulations is within the boundaries of $80 \%$ and $125 \%$.

The primary hypothesis of F/TAF's bioequivalence to E/C/F/TAF STR was tested using a Two One-Sided Tests (TOST) method with a 5\% significance level for each test. Approximately 104 evaluable subjects were estimated to conduct a TOST with $80 \%$ power for the $90 \%$ CI of the GLSM ratio of test vs. reference treatments with regard to AUC and $\mathrm{C}_{\max }$ to be contained within 0.80 and 1.25; this assumed a GLSM ratio of 1.05. If the true GLSM ratio was 1.0, the TOST with 104 evaluable subjects would have at least $98 \%$ power. A total enrollment of 116 subjects was planned for a $10 \%$ overage. SAS software (SAS Institute, Cary, North Carolina, USA) was used to perform the statistical summaries and analyses.

Subject demographic data, baseline characteristics, plasma concentrations, and $\mathrm{PK}$ parameters were summarized by treatment using descriptive statistics. For each analyte (FTC and TAF), the natural logarithmic transformation of $\mathrm{PK}$ parameters $\left(\mathrm{AUC}_{\text {last }}, \mathrm{AUC}_{\text {inf }}\right.$ and $\mathrm{C}_{\max }$ ) were compared between the test and reference treatments by an analysis of variance (ANOVA) using a mixed-effects model with treatment, period, and sequence as fixed effects and subject within sequence as a random effect. SAS ${ }^{\circ}$ PROC MIXED (SAS Institute, Cary, NC) code was used to calculate the treatment comparisons and corresponding $90 \%$ CIs. The safety analysis set included all randomized subjects who received at least 1 dose of study drug.

\section{Results}

\section{Subject demographics and disposition}

In Study 1472, evaluating F/TAF 200/10 mg, there were 100 subjects randomized and received at least 1 dose of study drug, and there were 116 subjects in Study 1473, evaluating F/TAF 200/25 mg. The majority of subjects was male (52\% and 66\%) and white (85\% and 53\%) in both studies, respectively. At baseline, the median age was 32 years (range: 18-45) and 33 (18-45), median BMI was $26.4 \mathrm{~kg} / \mathrm{m}^{2}$ (interquartile range [IQR]: 23.5-28.1) and 25.9 (24.0-27.9), and median creatinine clearance by Cockcroft-Gault method was $135.4 \mathrm{~mL} / \mathrm{min}$ (IQR: 116.8-150.2) and 112.2 (101.0-132.9) in the F/TAF 200/10 $\mathrm{mg}$ and 200/25 mg studies, respectively. Two subjects did not complete the F/TAF 200/10 mg study due to 1 subject having an adverse event leading to discontinuation and another subject withdrawing consent. All 116 F/TAF 200/25 mg subjects completed the study.

\section{Pharmacokinetics}

In both bioequivalence studies, the PK analysis sets of plasma FTC and TAF included all subjects who were randomized and received at least
1 dose of study drug. Mean (SD) FTC and TAF plasma concentrationtime profiles are presented in Figure 1 (F/TAF 200/10 mg) and Figure 2 (F/TAF 200/25 mg). In both studies, the plasma concentrations of FTC and TAF were similar after administration of the test or reference treatment throughout the monitoring period (Treatments A and B [144 hours]). Plasma PK parameters for FTC and TAF after administration of the test or reference treatment are presented in Table 1.

The statistical analyses of FTC and TAF PK parameters between test and reference treatments are presented in Table 2.The 90\% CIs for the GLSM ratios of the primary PK parameters $\mathrm{AUC}_{\text {last }}, \mathrm{AUC}_{\mathrm{inf}}$, and $\mathrm{C}_{\max }$ for test versus reference treatments were within the protocolspecified bioequivalence boundary of $80 \%$ to $125 \%$ for FTC and TAF, in both studies. FTC and TAF component of F/TAF FDCs, at both dose strengths, were bioequivalence to FTC and TAF component of E/C/F/ TAF.

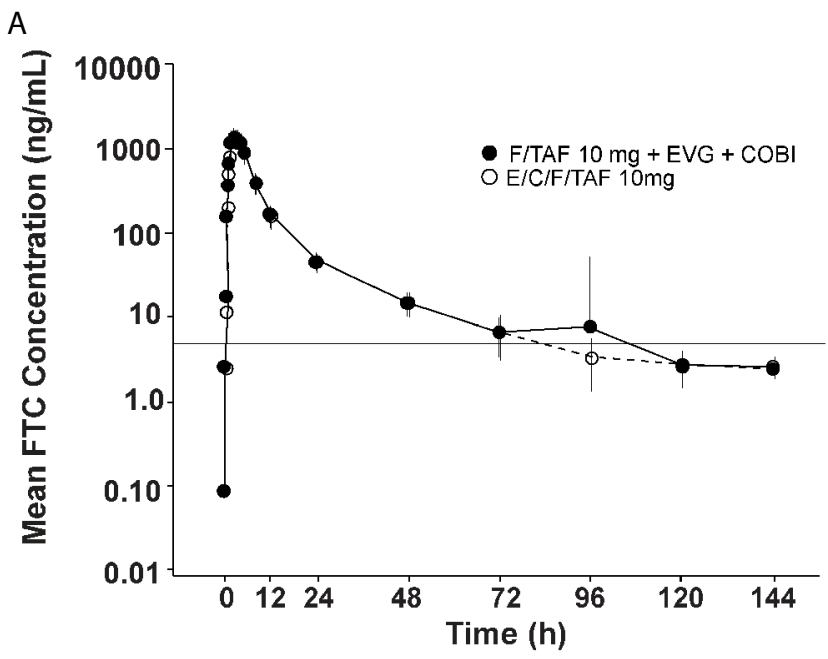

Figure 1A: Mean (SD) plasma concentration-time profile of FTC (N=97) after administration of Treatment A (test: $200 / 10 \mathrm{mg} \mathrm{FTC/TAF+EVG+COBI)}$ and Treatment B (reference: EVG/COBI/FTC/TAF).

B

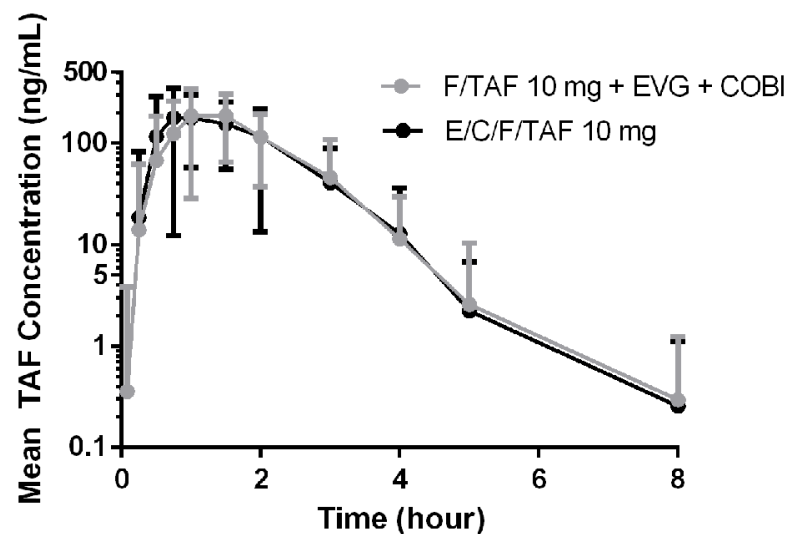

Figure 1B: Mean (SD) plasma concentration-time profile of TAF $(\mathrm{N}=97)$ after administration of Treatment $A$ (test: $200 / 10 \mathrm{mg} F T C / T A F+E V G+C O B I)$ and Treatment B (reference: EVG/COBI/FTC/TAF). 
Citation: Zack J, Chu H, Chuck S, Rhee M, Koziara J, et al. (2016) Bioequivalence of Two Co-formulations of Emtricitabine/Tenofovir Alafenamide Fixed-Dose Combinations with 200/10 mg and 200/25 mg. J Bioequiv Availab 8: 068-073. doi:10.4172/jbb.1000270

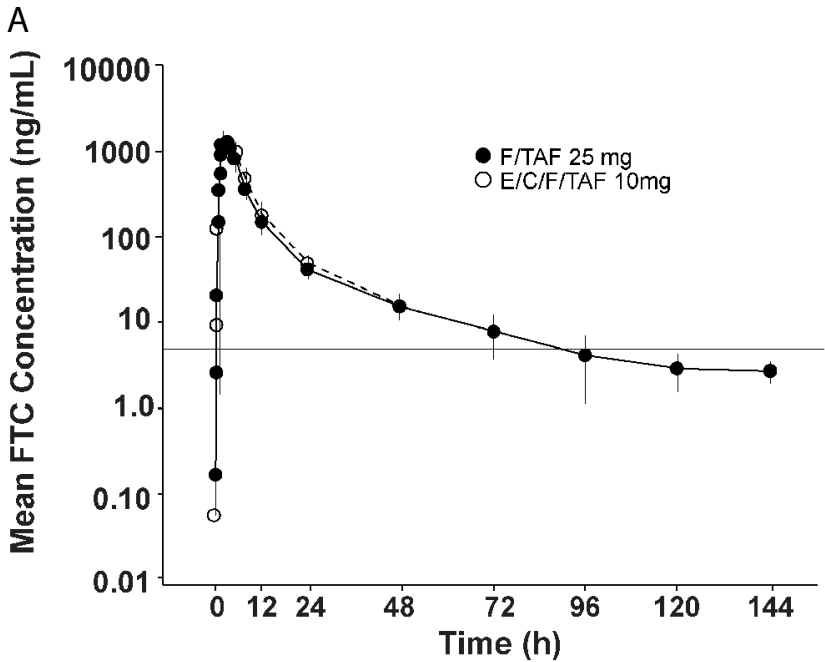

Figure 2A: Mean (SD) plasma concentration-time profile of FTC $(\mathrm{N}=116)$ after administration of Treatment A (test: $200 / 25 \mathrm{mg} \mathrm{FTC/TAF}$ ) and Treatment B (reference: EVG/COBI/FTC/TAF).
B

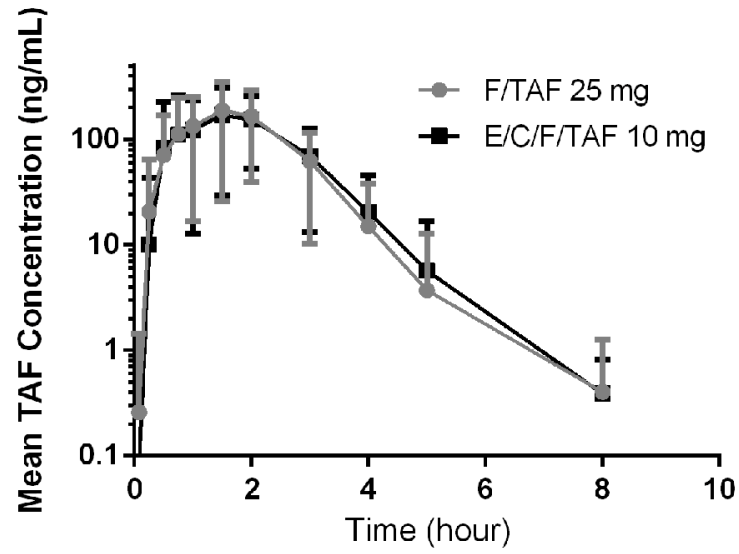

Figure 2B: Mean (SD) plasma concentration-time profile of TAF $(\mathrm{N}=116)$ after administration of Treatment A (test: $200 / 25 \mathrm{mg}$ FTC/TAF) and Treatment B (reference: EVG/COBI/FTC/TAF).

\begin{tabular}{|c|c|c|c|c|c|c|c|c|}
\hline \multirow[b]{3}{*}{$\begin{array}{l}\text { PK } \\
\text { Parameter }\end{array}$} & \multicolumn{4}{|c|}{ F/TAF $200 / 10$ mg $^{b}$} & \multicolumn{4}{|c|}{ F/TAF $200 / 25 \mathrm{mg}$} \\
\hline & \multicolumn{2}{|c|}{ FTC } & \multicolumn{2}{|c|}{ TAF } & \multicolumn{2}{|c|}{ FTC } & \multicolumn{2}{|c|}{ TAF } \\
\hline & $\begin{array}{c}\text { Test }-\mathrm{FTCl} \\
\mathrm{TAF}+\mathrm{EVG}+\mathrm{COBI} \\
(\mathrm{N}=97)\end{array}$ & $\begin{array}{c}\text { Reference- } \\
\text { EVG/COBI/FTC/ } \\
\text { TAF (N=99) }\end{array}$ & $\begin{array}{c}\text { Test }-\mathrm{FTC} / \mathrm{TAF}+ \\
\text { EVG+COBI } \\
(\mathrm{N}=97)\end{array}$ & $\begin{array}{c}\text { Reference - EVG/ } \\
\text { COBI/FTC/TAF } \\
(\mathrm{N}=99)\end{array}$ & $\begin{array}{c}\text { Test }-\mathrm{FTC} / \mathrm{TAF} \\
(\mathrm{N}=116)\end{array}$ & $\begin{array}{c}\text { Reference - EVG/ } \\
\text { COBI/FTC/TAF } \\
(\mathrm{N}=116)\end{array}$ & $\begin{array}{c}\text { Test }-\mathrm{FTC} / \mathrm{TAF} \\
(\mathrm{N}=116)\end{array}$ & $\begin{array}{c}\text { Reference - EVG/ } \\
\text { COBI/FTC/TAF } \\
(\mathrm{N}=116)\end{array}$ \\
\hline $\begin{array}{l}\mathrm{AUC}_{\text {inf }}(\mathrm{ng} \cdot \mathrm{h} / \\
\mathrm{mL})\end{array}$ & $10535.1(27.0)$ & $10294.4(15.8)$ & $351.8(31.0)^{c}$ & $354.1(32.9)^{d}$ & $9654.6(19.3)$ & $10706.6(19.6)$ & $396.4(42.6)$ & 389.5 (39.3) \\
\hline $\begin{array}{l}\mathrm{AUC}_{\text {last }}(\mathrm{ng} \cdot \mathrm{h} / \\
\mathrm{mL})\end{array}$ & $10159.2(17.2)$ & $10086.8(15.9)$ & $336.6(33.9)$ & $340.2(33.8)$ & $9423.9(19.3)$ & $10475.3(19.7)$ & $374.0(43.4)$ & $369.3(40.6)$ \\
\hline $\mathrm{C}_{\max }(\mathrm{ng} / \mathrm{mL})$ & $1660.8(20.6)$ & $1662.6(19.1)$ & $301.6(48.8)$ & $310.3(48.7)$ & $1577.4(26.8)$ & $1601.7(19.6)$ & $280.5(62.9)$ & $267.8(59.8)$ \\
\hline$t_{1 / 2}(h)$ & $18.1(46.8)$ & $19.1(57.0)$ & $0.41(39.5)^{c}$ & $0.43(35.4)^{d}$ & $22.3(52.0)$ & $21.9(55.6)$ & $0.5(27.1)^{\mathrm{e}}$ & $0.5(38.5)^{f}$ \\
\hline $\mathrm{T}_{\max }(\mathrm{h})$ & $2.0(1.5,3.0)$ & $2.0(1.5,3.0)$ & $1.5(1.0,1.5)$ & $1.0(0.8,2.0)$ & $2.0(2.0,3.0)$ & $3.0(2.0,3.0)$ & $1.5(1.0,2.0)$ & $1.5(1.0,2.0)$ \\
\hline
\end{tabular}

$A \cup C_{\text {inf }}=$ Area Under the Plasma Concentration Versus Time Curve Extrapolated to Infinity, AUC $C_{\text {last }}=$ Area Under the Plasma Concentration Versus Time Curve from Time 0 to the Last Quantifiable Concentration, $\mathrm{C}_{\max }=$ Maximum Observed Plasma Concentration of Drug, $\mathrm{t}_{1 / 2}=$ Elimination Half-Life of the Drug in Plasma, $T_{\text {max }}=$ Time to Maximum Concentration

a. Data are mean (\%CV) except $T_{\max }$, which is reported as median (Q1, Q3).

b. Two subjects in Test and 1 subject in Reference were excluded from the primary analysis due to incomplete blood draw data on Day 1.

c. $\mathrm{n}=80$

d. $n=84$

e. $n=95$

f. $n=97$

Table 1: Summary of FTC and TAF pharmacokinetic parameters.

\section{Safety}

In both bioequivalence studies, all treatments were generally well tolerated by the study subjects. There were no deaths or Grade 4 AEs reported in either study. The one serious Grade $3 \mathrm{AE}$ of peritoneal hemorrhage was reported with F/TAF 200/25 mg and considered by the investigator as not related to the study drug. No laboratory abnormalities were considered clinically significant in either study. In the F/TAF 200/25 mg bioequivalence Study 1473, one subject had Grade 4 lipase, but did not exhibit clinical symptoms. In both bioequivalence studies, no clinically relevant changes in median values for hematology and chemistry parameters were observed.

In the F/TAF 200/10 mg bioequivalence Study 1472, the majority of AEs were Grade 1 in severity with one case of Grade 3 arthralgia, no Grade $4 \mathrm{AEs}$, and only one subject discontinued therapy due to a treatment-related AE - Grade 2 macular rash with E/C/F/TAF. Treatment-related Grade 2 AEs reported by more than 1 subject consisted of only headache -3 subjects with F/TAF 200/10 mg+EVG+COBI and 4 subjects with $\mathrm{E} / \mathrm{C} / \mathrm{F} / \mathrm{TAF}$. The most frequently reported AEs $(>2.5 \%)$ with F/TAF 200/10 mg+EVG+COBI were headache and nausea $(9.1 \%$ each); diarrhea (4.0\%); pruritus, papular rash, feeling hot, and anxiety (3.0\% each) and with E/C/F/TAF were headache (10.0\%) and nausea (6.0\%).

In the F/TAF 200/25 mg bioequivalence Study 1473, the majority of AEs were Grade 1 in severity with no Grade 4 AEs reported. The only treatment-related Grade 2 AEs reported was nausea and vomiting in 1 subject with $\mathrm{E} / \mathrm{C} / \mathrm{F} / \mathrm{TAF}$. The most frequently reported AEs with $\mathrm{F} /$ TAF $200 / 25 \mathrm{mg}$ were nausea (4.3\%), constipation, and headache $(2.6 \%$ each) and with $\mathrm{E} / \mathrm{C} / \mathrm{F} / \mathrm{TAF}$ were nausea (5.2\%), vomiting, and headache (2.6\% each).

\section{Discussion}

The results of the bioequivalence Study 1472 demonstrate that 


\begin{tabular}{|c|c|c|c|c|c|c|c|c|}
\hline & \multicolumn{4}{|c|}{ FTC/TAF $200 / 10 \mathrm{mg}$} & \multicolumn{4}{|c|}{ FTC/TAF $200 / 25 \mathrm{mg}$} \\
\hline & \multicolumn{2}{|l|}{ FTC } & \multicolumn{2}{|l|}{ TAF } & \multicolumn{2}{|c|}{ FTC } & \multicolumn{2}{|c|}{ TAF } \\
\hline & $\begin{array}{l}\text { GLSM Ratio (Test/ } \\
\text { Reference) }(\%)\end{array}$ & $90 \% \mathrm{Cl}(\%)$ & $\begin{array}{l}\text { GLSM Ratio (Test/ } \\
\text { Reference) (\%) }\end{array}$ & $90 \% \mathrm{Cl}(\%)$ & $\begin{array}{l}\text { GLSM Ratio (Test/ } \\
\text { Reference) }(\%)\end{array}$ & $90 \% \mathrm{Cl}(\%)$ & $\begin{array}{l}\text { GLSM Ratio (Test/ } \\
\text { Reference) (\%) }\end{array}$ & $90 \% \mathrm{Cl}(\%)$ \\
\hline$A \cup C_{\text {last }}(\mathrm{ng} \cdot \mathrm{h} / \mathrm{mL})$ & 99.8 & $98.4,101.3$ & 98.0 & $94.7,101.3$ & 90.0 & $88.9,91.2$ & 100.3 & $96.5,104.3$ \\
\hline $\mathrm{AUC}_{\mathrm{inf}}(\mathrm{ng} \cdot \mathrm{h} / \mathrm{mL})$ & 100.7 & $98.2,103.2$ & 98.3 & $94.8,102.0$ & 90.2 & $89.1,91.4$ & 98.5 & $94.6,102.6$ \\
\hline $\mathrm{C}_{\max }(\mathrm{ng} / \mathrm{mL})$ & 99.6 & $96.8,102.4$ & 96.9 & $89.4,105.0$ & 97.3 & $94.6,100.0$ & 103.6 & $95.5,112.5$ \\
\hline
\end{tabular}

Table 2: Statistical comparisons of FTC and TAF PK parameters for test vs. reference treatments.

under fed conditions F/TAF 200/10 mg administered simultaneously with EVG and COBI is bioequivalent to E/C/F/TAF STR. Furthermore, the results of the bioequivalence Study 1473 demonstrate that under fed conditions F/TAF 200/25 mg, without a PK enhancer, is bioequivalent to the FTC and TAF components of E/C/F/TAF STR that includes a $\mathrm{PK}$ enhancer. The selection of the TAF dose (10 or $25 \mathrm{mg})$ is based on whether or not the third ARV agent requires a PK enhancer (i.e., COBI or RTV) that results in increased TAF absorption due to the inhibition of intestinal p-glycoprotein-mediated efflux of TAF [9]. Specifically, F/TAF $200 / 25 \mathrm{mg}$ is recommended with third agents that do not have clinically relevant effect on TAF exposure (e.g., efavirenz [EFV], rilpivirine [RPV], dolutegravir [DTG]). F/TAF 200/10 mg is recommended with third agents that substantially increase TAF exposure (e.g., atazanavir+RTV, lopinavir/RTV, EVG+COBI [as $\mathrm{E} / \mathrm{C} / \mathrm{F} / \mathrm{TAF}])$. The development of these two once daily F/TAF FDCs is supported by these critical bioequivalence findings as well as the long intracellular half-lives of FTC-triphosphate ( $\sim 39$ hours) and TFV-DP ( 150 hours) [10,11].

Formal bioequivalence evaluations are an essential part of the regulatory approval strategy in the development of FDCs when supportive clinical trial data of the components exists. This strategy expedites the approval process since clinical evaluations with the FDC can continue after the submission of the marketing application to the proper regulatory authorities. To date, 3 of the 4 available antiretroviral STRs (EFV/FTC/TDF [Atripla], RPV/FTC/TDF [Complera/Eviplera], and DTG/lamivudine/abacavir [Triumeq]) have been approved by regulatory authorities for the treatment of HIV-1 infection based on such a bioequivalence strategy that compared the PK of the test coformulation to the PK of the approved antiretroviral components [12-14]. The two bioequivalence studies presented here are unique in that they bridge the safety and efficacy data of the approved $\mathrm{E} / \mathrm{C} / \mathrm{F} /$ TAF STR, evaluated in numerous registrational trials, to the NRTI components of the FDC, F/TAF, at two different doses.

The E/C/F/TAF clinical development program is the largest to date for an antiretroviral, consisting of 23 clinical trials with $>3000$ subjects, and has led to the approval of E/C/F/TAF in November 2015 by the US Food and Drug Administration. In the pooled analysis of two randomized, double-blind, phase 3 , non-inferiority studies in treatment-naïve, HIV-infected adults, E/C/F/TAF demonstrated non-inferior efficacy to $\mathrm{E} / \mathrm{C} / \mathrm{F} / \mathrm{TDF}$ at Week 48 , significantly less proteinuria, no discontinuations due to renal AEs, and significantly less decrease in spine and hip BMD [3,15]. Similarly, in two phase 3 studies of virologically suppressed adults who switched off other antiretroviral regimens, E/C/F/TAF maintained high rates of virologic suppression at Week 48 and resulted in significant improvement in proteinuria, no cases of proximal renal tubulopathy or Fanconi syndrome, and increases in spine and hip BMD [16,17]. Since the two bioequivalence studies reported here found the plasma tenofovir alafenamide exposures were bioequivalent for 1) F/TAF 200/10 mg FDC with a PK booster (simultaneous dosing of COBI tablet and EVG tablet) compared to F/ TAF 200/10 $\mathrm{mg}$ in the form of E/C/F/TAF STR and 2) F/TAF 200/25 mg
FDC without a PK enhancer compared to F/TAF 200/10 mg in the form of E/C/F/TAF STR, both FTC/TAF FDCs would be expected to have a $>90 \%$ decrease in plasma tenofovir exposure similar to that reported in the pooled Phase 3 treatment-naive studies of E/C/F/TAF [3]. This significant reduction in plasma tenofovir exposures is felt to explain the reduced off-target effects of tenofovir on the bone and kidneys observed in the clinical trials of TAF [3,15-17].

In addition to an improved renal and bone safety profile, TAF offers several advantages over abacavir including decreased cardiovascular risk, no risk of hypersensitivity reaction, no need for HLA testing, and less reproductive risk (pregnancy category B). As a FDC, F/TAF will decrease pill burden while giving clinicians the flexibility to tailor the third ARV agent to the needs of the patients. F/TAF will also be substantially smaller than the current FTC/TDF tablet given the reduction in the dose of the TFV prodrug ( $300 \mathrm{mg}$ vs. $25 \mathrm{mg}$ or $10 \mathrm{mg}$ ) with FTC/TDF measuring $19 \mathrm{~mm} \times 8.5 \mathrm{~mm} \times 7 \mathrm{~mm}$ and both F/TAF formulations measuring $12.5 \mathrm{~mm} \times 6.4 \mathrm{~mm} \times 4.3 \mathrm{~mm}$, respectively. Both F/TAF FDCs address pill size and dosing frequency, which are two important attributes recognized by patients to impact their adherence [18]. Besides the immediate anticipated advantages, F/TAF has led to the development of new STRs, with third agents such as EVG/COBI, $\mathrm{RPV}$, and darunavir/COBI, because there is substantially less active pharmaceutical ingredient for TAF vs. TDF. Furthermore, drug-drug interaction studies have been conducted showing F/TAF-based products including $\mathrm{E} / \mathrm{C} / \mathrm{F} / \mathrm{TAF}$ and $\mathrm{R} / \mathrm{F} / \mathrm{TAF}$ STRs can be co-administered safely with ledipasvir/sofosbuvir without dose adjustment [19].

Evaluations of drug interactions between TAF and various third agents have been completed to inform the TAF dose. These studies have included atazanavir, darunavir, dolutegravir, lopinavir, and rilpivirine [20,21]. These data confirmed that TAF $10 \mathrm{mg}$ should be used in regimens containing a RTV- or COBI-boosted protease inhibitor and TAF $25 \mathrm{mg}$ should be used in regimens with other agents, such as integrase strand transfer inhibitors (INSTIs) and non-nucleoside reverse transcriptase inhibitor (NNRTIs). Three on-going phase 3 studies of F/TAF allow the following common third agents: 3 NNRTIs (EFV, NVP, RPV), 2 INSTIs (dolutegravir, raltegravir), 3 PIs (RTV- or COBI-boosted atazanavir, RTV- or COBI-boosted darunavir, lopinavir/ RTV), and maraviroc [clinicaltrials.gov NCT02121795, NCT02469246, and NCT02285114].

\section{Conclusion}

In conclusion, F/TAF 200/10 mg FDC administered simultaneously with EVG and COBI and F/TAF 200/25 mg FDC administered without a PK enhancer are bioequivalent to the E/C/F/TAF STR allowing for extrapolation of the safety and efficacy data generated in the registrational trials with $\mathrm{E} / \mathrm{C} / \mathrm{F} / \mathrm{TAF}$. It is anticipated that these bioequivalence results will support the registration of the F/TAF 200/10 mg and 200/25 mg FDCs as the next advancement in NRTI backbone, emphasizing safety while continuing the history of efficacy, convenience, and tolerance for treatment of HIV-1 infection. 
Citation: Zack J, Chu H, Chuck S, Rhee M, Koziara J, et al. (2016) Bioequivalence of Two Co-formulations of Emtricitabine/Tenofovir Alafenamide Fixed-Dose Combinations with 200/10 mg and 200/25 mg. J Bioequiv Availab 8: 068-073. doi:10.4172/jbb.1000270

\section{References}

1. Department for Health and Human Services (DHHS) (2015) Guidelines for the use of antiretroviral agents in HIV-1-infected adults and adolescents. Developed by the DHHS panel on antiretroviral guidelines for adults and adolescents - A working group of the office of AIDS research advisory council (OARAC).

2. Gilead Sciences Inc. VIREAD ${ }^{\circledR}$ (tenofovir disoproxil fumarate) tablets, for ora use. US Prescribing Information. Revised May 2015

3. Sax PE, Wohl D, Yin MT, Post F, DeJesus E, et al. (2015) Tenofovir alafenamide versus tenofovir disoproxil fumarate, coformulated with elvitegravir, cobicistat, and emtricitabine, for initial treatment of HIV-1 infection: two randomised, double-blind, phase 3, non-inferiority trials. Lancet 385: 2606-2615.

4. Ruane PJ, DeJesus E, Berger D, Markowitz M, Bredeek UF, et al. (2013) Antiviral activity, safety, and pharmacokinetics/pharmacodynamics of tenofovir alafenamide as 10-day monotherapy in HIV-1-positive adults. J Acquir Immune Defic Syndr 63: 449-455

5. Gelder JV, Deferme S, Naesens L, Clercq ED, Mooter GVD, et al. (2002) Intestinal absorption enhancement of the ester prodrug tenofovir disoproxil fumarate through modulation of the biochemical barrier by defined ester mixtures. Drug Metab Dispos 30: 924-930.

6. Babusis D, Phan TK, Lee WA, Watkins WJ, Ray AS (2013) Mechanism for effective lymphoid cell and tissue loading following oral administration of nucleotide prodrug GS-7340. Mol Pharm 10: 459-466.

7. Tong L, Phan TK, Robinson KL, Babusis D, Strab R, et al. (2007) Effects of human immunodeficiency virus protease inhibitors on the intestinal absorption of tenofovir disoproxil fumarate in vitro. Antimicrob Agents Chemother 51 : 3498-3504.

8. Lepist El, Phan TK, Roy A, Tong L, MacLennan K, et al. (2012) Cobicista boosts the intestinal absorption of transport substrates, including HIV protease inhibitors and GS-7340, in vitro. Antimicrob Agents Chemother 56: 5409-5413.

9. Custodio JM, Shao Y, Wei X, Vu A, Rhee M, et al. (2015) The effect of food on the pharmacokinetics of unboosted and boosted tenofovir alafenamide [Poster PE10/13]. 15 ${ }^{\text {th }}$ European AIDS Conference (EACS); 21-24 October, Barcelona, Spain.

10. Hawkins T, Veikley W, St Claire RL $3^{\text {rd }}$, Guyer B, Clark N, et al. (2005) Intracellular pharmacokinetics of tenofovir diphosphate, carbovir triphosphate, and lamivudine triphosphate in patients receiving triple-nucleoside regimens. $J$ Acquir Immune Defic Syndr 39: 406-411.

11. Molina JM, Cox SL (2005) Emtricitabine: a novel nucleoside reverse transcriptase inhibitor. Drugs Today (Barc) 41: 241-252.
12. Mathias AA, Hinkle J, Menning M, Hui K, Kaul S, et al. (2007) Bioequivalence of efavirenz/emtricitabine/tenofovir disoproxil fumarate single-tablet regimen. $J$ Acquir Immune Defic Syndr 46: 167-173.

13. Mathias A, Menning M, Wiser L, Wei X, Dave A, et al. (2012) Bioequivalence of the emtricitabine/rilpivirine/tenofovir disoproxil fumarate single tablet regimen. J Bioequiv Availab 4: 100-105.

14. Weller S, Chen S, Borland J, Savina P, Wynne B, et al. (2014) Bioequivalence of a dolutegravir, abacavir, and lamivudine fixed-dose combination tablet and the effect of food. J Acquir Immune Defic Syndr 66: 393-398.

15. Wohl D, Oka S, Clumeck N, Clarke A, Brinson C, et al. (2015) A randomized double-blind comparison of tenofovir alafenamide vs tenofovir disoproxi fumarate, each coformulated with elvitegravir, cobicistat, and emtricitabine, for initial HIV-1 treatment: Week 96 results [Poster LBBPD1/1]. 15 $5^{\text {th }}$ European AIDS Conference (EACS); 21-24 October, Barcelona, Spain.

16. Mills A, Arribas JR, Andrade-Villanueva J, DiPerri G, Van Lunzen J, et al. (2015) Switching from tenofovir disoproxil fumarate to tenofovir alafenamide in antiretroviral regimens for virologically suppressed adults with HIV-1 infection: a randomised, active-controlled, multicentre, open-label, phase 3 , non-inferiority study. Lancet Infect Dis 16: 43-52.

17. Pozniak A, Arribas J, Gupta SK, Post FA, Avihingsanon A, et al. (2015) Safety of tenofovir alafenamide in renal impairment [Poster 795]. Conference on Retroviruses and Opportunistic Infections (CROI); 23-26 February, Seattle, WA

18. Stone VE, Jordan J, Tolson J, Miller R, Pilon T (2004) Perspectives on adherence and simplicity for HIV-infected patients on antiretroviral therapy: Self-report of the relative importance of multiple attributes of highly active antiretroviral therapy (HAART) regimens in predicting adherence. J Acquir Immune Defic Syndr 36: 808-816.

19. Custodio JM, Doyle EB, Pang PS, Das M, Cao H, et al. (2015) Lack of drug interactions between boosted and unboosted TAF-based antiretroviral singletablet regimens (E/C/F/TAF, R/F/TAF) and the anti-HCV single-tablet regimen ledipasvir/sofosbuvir [Presentation 747]. IDWeek; 7-11 October, San Diego CA

20. Lawson EB, Martin H, McCallister S, Shao Y, Vimal M, et al. (2014) Drug interactions between tenofovir alafenamide and HIV antiretroviral agents [Poster H-1012]. 54 ${ }^{\text {th }}$ Interscience Conference on Antimicrobial Agents and Chemotherapy (ICAAC); 5-9 September, Washington DC, USA.

21. Custodio JM, Wang H, Silva A, Zhong L, Zack JZ, et al. (2015) Drug interaction potential of emtricitabine/tenofovir alafenamide fixed dose combination and cobicistat-boosted darunavir [Poster PII-044]. American Society for Clinical Pharmacology and Therapeutics (ASCPT); 3-7 March, New Orleans, LA. 